\title{
Fotossíntese foliar do capim-xaraés [Brachiaria brizantha (A. Rich.) Stapf. cv. Xaraés] e modelagem da assimilação potencial de dosséis sob estratégias de pastejo rotativo
}

\author{
Bruno Carneiro e Pedreira ${ }^{1}$, Carlos Guilherme Silveira Pedreira ${ }^{1}$
}

${ }^{1}$ USP/ESALQ.

RESUMO - Objetivou-se com este trabalho quantificar a fotossíntese foliar ao longo do período de rebrotação e modelar a fotossíntese de dosséis vegetativos em função da arquitetura foliar e do ambiente luminoso em pastagens de capim-xaraés [Brachiaria brizantha (A. Rich.) Stapf.] submetidas a estratégias contrastantes de pastejo rotativo. Na fase inicial de rebrotação, o potencial fotossintético foi pequeno, em média $10,4 \mu \mathrm{mol} \mathrm{CO}_{2} \mathrm{~m}^{-2} \mathrm{~s}^{-1}$ e, no tratamento de maior intervalo de pastejo $(100 \% \mathrm{IL})$, esse potencial foi ainda menor $\left(3,3 \mu \mathrm{mol} \mathrm{CO}_{2} \mathrm{~m}^{-2} \mathrm{~s}^{-1}\right)$, o que pode comprometer o desenvolvimento das plantas. No dia médio (DM) do ciclo, ocorreram os maiores valores de fotossíntese foliar, em média $26 \mu \mathrm{mol} \mathrm{CO}_{2} \mathrm{~m}^{-2} \mathrm{~s}^{-1}$. No pré-pastejo, o tratamento $95 \%$, que apresentou os menores intervalos de pastejo (22 dias), continuou com os maiores valores de fotossíntese foliar $\left(26,1 \mu \mathrm{mol} \mathrm{CO}_{2} \mathrm{~m}^{-2} \mathrm{~s}^{-1}\right)$. Enquanto isso, os tratamentos com maiores intervalos de pastejo (28 e 32 dias, respectivamente para o tratamento 28 dias e $100 \%$ IL) tiveram seus valores de fotossíntese foliar reduzidos (21,3 e $24,5 \mu \mathrm{mol} \mathrm{CO} \mathrm{CO}_{2} \mathrm{~m}^{-2} \mathrm{~s}^{-1}$ ). No tratamento de maior freqüência de pastejo (95\% de IL), o menor intervalo de pastejo e a manutenção de menor IAF médio $(2,08)$ resultaram em maior entrada de luz no interior do dossel. O manejo adequado do capim-xaraés deve ser feito com intervalos de pastejo inferiores a 28 dias, a fim de possibilitar maiores valores de fotossíntese de dossel ao longo do ciclo de rebrotação.

Palavras-chave: assimilação de carbono, coeficiente de extinção luminosa, fotossíntese de dossel, índice de área foliar

\section{Leaf photosynthesis in Xaraés palisadegrass [Brachiaria brizantha (A. Rich.) Stapf. cv. Xaraés] and modeling canopy assimilatory potential under rotational stocking strategies}

\begin{abstract}
The objective of this research was to quantify leaf photosynthetic rates during the regrowth period and to model canopy photosynthesis of vegetative swards of Xaraés palisadegrass [Brachiaria brizantha (A. Rich.) Stapf.] under contrasting management strategies of rotational stocking. Early regrowth was characterized by low photosynthetic potential $\left(10.4 \mu \mathrm{mol} \mathrm{CO} \mathrm{m}^{-2} \mathrm{~s}^{-1}\right)$ and for paddocks under the $100 \%$ LI treatment, leaf photosynthesis was $3.3 \mu \mathrm{mol}$ $\mathrm{CO}_{2} \mathrm{~m}^{-2} \mathrm{~s}^{-1}$, which may require plants to draw on organic reserves during the early phases of the process. Half way through regrowth, leaf photosynthetic rates increased to $26 \mu \mathrm{mol} \mathrm{CO} \mathrm{m}^{-2} \mathrm{~s}^{-1}$ in all treatments. Paddocks under the $95 \% \mathrm{LI}$ treatment (shortest mean regrowth periods; 22 days), sustained pre-graze photosynthetic rates at $26.1 \mu \mathrm{mol} \mathrm{CO}_{2} \mathrm{~m}^{-2} \mathrm{~s}^{-1}$. The longer regrowth intervals (28 and 32 days on average, for the $28 \mathrm{~d}$ and for the $100 \%$ LI paddocks, respectively), resulted in lower rates of 21.3 and $24.5 \mu \mathrm{mol} \mathrm{CO} \mathrm{m}^{-2} \mathrm{~s}^{-1}$, respectively. Under the highest grazing frequency $(95 \% \mathrm{LI})$, the short rest period and the low mean LAI (2.08) allowed for more light penetration into the canopy. Optimum management practices for Xaraés palisadegrass should include grazing intervals shorter than 28 days, so that higher photosynthetic rates are achieved during the regrowth period.
\end{abstract}

Key Words: canopy photosynthesis, carbon assimilation, leaf area index, light extinction coefficient

\section{Introdução}

Cooper \& Wilson (1970) postularam que a eficiência do dossel na conversão da energia luminosa em MS depende das taxas de fotossíntese de folhas individuais e dos padrões de interceptação luminosa (IL) do dossel. O acúmulo de MS em plantas forrageiras é resultante de interações complexas de atributos genéticos e de ambiente e seus efeitos sobre os processos fisiológicos e sobre as características morfológicas das plantas (Da Silva \& Pedreira, 1997).

Segundo Bernardes (1987), vários aspectos morfofisiológicos estão envolvidos na interceptação da luz pelas plantas em comunidade. Uns correspondem a aspectos relacionados 
à organização espacial das folhas, que pode ser expressa pela densidade de cobertura foliar, pela distribuição horizontal e vertical das folhas e pelos ângulos foliares. Outros correspondem a aspectos funcionais que dependem de fatores da planta e do ambiente, como idade, tipo e tamanho das folhas, saturação lumínica e flutuações na intensidade e na qualidade de luz. Verhagen et al. (1963) verificaram que a produção de MS depende fundamentalmente da eficiência das folhas em utilizar a luz incidente e de como essa luz é distribuída ao longo do dossel. Dessa forma, a utilização da energia solar é influenciada por alguns fatores, como propriedades óticas das folhas, intensidade de luz e distribuição espacial das folhas. Verhagen et al. (1963) e Pearce et al. (1965) demonstraram que a produção de forragem depende, em última análise, do uso da luz interceptada pelo dossel.

$\mathrm{O}$ aumento da fitomassa em uma área cultivada depende do desenvolvimento de sua área foliar. Segundo Loomis \& Williams (1969), a arquitetura do dossel interfere tanto na distribuição da luz dentro da população de plantas como na circulação de ar e ainda afeta os processos de transferência de $\mathrm{CO}_{2}$ e evapotranspiração. Portanto, a arquitetura do dossel vegetativo é determinante dos padrões de interceptação luminosa pelas plantas e, provavelmente, uma das características mais importantes que determina sua habilidade. Pequenas diferenças em altura podem ter grandes efeitos na competição por luz, pois uma diferença mínima é suficiente para uma folha se sobrepor a outra (Loomis \& Williams, 1969).

Quando os intervalos de desfolhações são curtos, plantas com maior proporção do IAF na parte inferior do dossel apresentam maior IAF residual, o que assegura rápida rebrotação inicial após desfolhação, em decorrência da maior interceptação luminosa. Entretanto, se o período de rebrotação é longo, as plantas de crescimento mais alto e ereto, com maiores proporções do IAF nas regiões intermediária e superior do dossel, têm tempo suficiente para acumular um grande IAF e utilizam melhor a radiação incidente, tornando-se, portanto, mais produtivas (Rhodes, 1973). À medida que o índice de área foliar aumenta, ocorre decréscimo na penetração de luz até o nível do solo durante o crescimento de uma cultura (Brown, 1984).

Folhas individuais apresentam grande variação em sua capacidade fotossintética, determinada por vários fatores, como quantidade de radiação solar incidente, temperatura, suprimento de água e, principalmente, o estádio de desenvolvimento da folha (Zelich, 1982; Braga et al., 2006; Pedreira et al., 2001). Ao longo do perfil vertical de um dossel, as variações de temperatura e luminosidade e a diferença na idade fisiológica das folhas são grandes, o que lhes confere diferentes potenciais fotossintéticos (Loomis \& Williams, 1969).

Modelos matemáticos podem ser utilizados para prever a fotossíntese do dossel (Jonhson et al., 1989). As respostas agronômicas de espécies forrageiras ao manejo e ao ambiente podem ser preditas de forma a melhorar as estratégias de manejo de colheita de forragem, seja por corte seja por pastejo (Rymph, 2004). Na otimização do sistema de produção animal, é necessário o conhecimento de aspectos morfofisiológicos das plantas forrageiras associados aos efeitos da ação do animal sobre a rebrotação. Estratégias de pastejo afetam as características da forragem e a utilização da IL como guia de manejo permite que as plantas sejam colhidas em condição fisiológica semelhante. Contrastar esta técnica à utilização de uma estratégia de pastejo baseada em tempo cronológico pode permitir quantificar as divergências entre os métodos. Diferentes freqüências de pastejo geram mudanças na estrutura do dossel, alterando o ambiente luminoso e resultando em dosséis com diferentes potenciais fotossintéticos.

Os objetivos neste trabalho foram quantificar a fotossíntese foliar ao longo do período de rebrotação e modelar a fotossíntese de dosséis vegetativos em relação à arquitetura foliar e ao ambiente luminoso em pastagens de capim-xaraés submetidas a estratégias contrastantes de pastejo rotativo, com freqüências de desfolhação determinadas pela interceptação luminosa ou pelo calendário.

\section{Material e Métodos}

O experimento foi conduzido em uma área experimental pertencente ao Departamento de Zootecnia da USP/ESALQ, em Piracicaba-SP, a $580 \mathrm{~m}$ de altitude, $22^{\circ} 42^{\prime}$ 'de latitude sul e $47^{\circ} 30^{\prime}$ 'de longitude oeste, e com características médias das normais meteorológicas correspondentes a uma precipitação de $1.002 \mathrm{~mm}$ e 23, $6^{\circ} \mathrm{C}$ de temperatura média durante o verão (outubro a março). O solo da área experimental é classificado como Nitossolo Vermelho Eutroférrico típico (Embrapa, 1999), sem necessidade de correção.

Foram feitas apenas adubações de produção, totalizando $120 \mathrm{~kg} \mathrm{~N} / \mathrm{ha}$ e $100 \mathrm{~kg} \mathrm{~K} / \mathrm{ha}$ nas formas de $\left(\mathrm{NH}_{4}\right)_{2} \mathrm{SO}_{4}$ e KCl, respectivamente. Foram realizadas duas adubações manuais, metade no início da estação (23/09/05) após pastejo uniforme, e o restante no meio do ciclo, entre os dias 3 e 16 de dezembro de 2005 , em condição póspastejo.

Durante os 153 dias do período experimental ( 22 de setembro de 2005 a 22 de fevereiro de 2006), os nove piquetes $\left(120 \mathrm{~m}^{2}\right)$ foram pastejados de acordo com o 
cronograma específico de cada tratamento (com base em IL ou no calendário), deixando-se um resíduo de $15 \mathrm{~cm}$, de acordo com a recomendação da Embrapa (Brasil, 2005) para o capim-xaraés.

Os tratamentos corresponderam a três estratégias de pastejo, todas de lotação rotacionada: duas definidas considerando a interceptação luminosa (IL) pelo dossel durante a rebrotação ( 95 e 100\% para início do pastejo) e uma baseada no tempo cronológico (pastejo a cada 28 dias). O experimento seguiu um delineamento inteiramente casualizado, com três repetições. A área de $1.080 \mathrm{~m}^{2}$ foi dividida em nove unidades experimentais (piquetes), cada uma com $120 \mathrm{~m}^{2}$. O pastejo foi feito por vacas das raças Holandesa (Bos taurus taurus L.) e Nelore (Bos taurus indicus L.) com peso médio de $350 \mathrm{~kg}$. A técnica de mobgrazing foi utilizada para a realização dos pastejos, utilizando-se grupos de animais para desfolhações rápidas (duração de 4 a 20 horas), simulando um cenário de pastejo rotativo. Cada piquete foi cercado com cerca elétrica para a contenção dos animais durante o pastejo. À medida que os animais pastejavam, foram feitas medições de altura até o dossel atingir, em média, $15 \mathrm{~cm}$ de altura.

As medições de interceptação luminosa (IL) e índice de área foliar (IAF) foram feitas durante dois ciclos de pastejo para cada piquete durante o verão agrostológico (16 de novembro a 20 de dezembro e de 17 de janeiro a 13 de fevereiro) e iniciaram imediatamente após o pastejo, a cada sete dias e imediatamente antes do pastejo seguinte, objetivando caracterizar as variações estruturais do dossel. Para isso, foi utilizado um analisador de dossel LI-COR modelo LAI 2000 (LI-COR, Lincoln Nebraska, EUA), cujo funcionamento foi descrito por Welles \& Normam (1991) e que permite amostragens rápidas e não-destrutivas. Esse aparelho é constituído de uma unidade de controle e de um sensor em formato de barra articulada. Na ponta da barra, existe um conjunto de lentes, tipo 'olho de peixe', que projetam a imagem hemisférica de baixo para cima do dossel, por meio de detetores de sílica. A técnica combina medidas tomadas com o sensor acima do dossel (Io) com medidas tomadas sob o dossel, próximas ao nível do solo (I). A partir dessas medidas, a inversão de um modelo de transferência de luz permite o cálculo do IAF (Welles \& Normam, 1991). Foram tomados 20 pontos representativos da condição média do dossel em cada piquete nas ocasiões de amostragem, na proporção de uma medida acima para cinco medidas abaixo do dossel, em quatro repetições, sempre utilizando estações entre touceiras. O tratamento $100 \%$ de IL, em virtude da impossibilidade prática de esse valor ser alcançado, foi assumido quando o dossel apresentava valores de interceptação luminosa superiores a $97,5 \%$ por dois dias consecutivos, pois, mesmo quando colocado em ausência total de luz, o aparelho (LAI-2000) não registra $100 \%$ de interceptação de luz.

As taxas de fotossíntese líquida de folhas individuais foram medidas nos mesmos dois ciclos em que IAF e IL foram caracterizados utilizando-se um sistema medidor de fotossíntese portátil, modelo LI-6400 (LI-COR, Lincoln Nebraska, EUA). As medições foram feitas em três momentos durante a rebrotação: imediatamente após o pastejo (dia seguinte à saída dos animais), imediatamente antes do pastejo subseqüente (dia imediatamente antes da entrada dos animais) e no dia médio do ciclo de rebrotação (DM). Para o tratamento baseado em calendário ( 28 dias), o dia médio foi o dia 14, e, para os tratamentos baseados em interceptação luminosa, o DM foi predito da seguinte forma: depois de terem sido feitas duas medições de IL, em um período de tempo conhecido, gerou-se uma taxa média de incremento na IL (unidades percentuais por dia), o que permitiu estimar o número de dias para atingir o tratamento (95 ou 100\% de IL) e, assim, determinar previamente o DM dos ciclos baseados em IL. Foram medidas as taxas de seis folhas em cada piquete, seguindo um critério de avaliação visual de modo a selecionar as melhores folhas presentes (a mais jovem completamente expandida, com mínimo de limbo, verdes e limpas), entre 8 e $11 \mathrm{~h}$, em dias de céu claro. Os perfilhos foram escolhidos em touceiras com altura semelhante à média do dossel na unidade experimental. A intensidade de luz na câmara foliar foi de $2.000 \mu \mathrm{mol}$ fótons $\mathrm{m}^{-2} \mathrm{~s}^{-1} \mathrm{e}$ foi utilizada concentração média de $\mathrm{CO}_{2}$ de $350 \mu \mathrm{mol} \mathrm{mol}^{-1}$, obtida com utilização de cilindros de $\mathrm{CO}_{2}$ acoplados ao aparelho. Nas medições de fotossíntese foliar realizadas pós-pastejo, nem sempre foi possível utilizar a folha mais jovem completamente expandida e intacta, principalmente em piquetes submetidos à estratégia de menor freqüência ( $100 \%$ de IL), em razão da ausência desse tipo de folhas.

Para estimativa da taxa de fotossíntese do dossel, utilizou-se o modelo de assimilação de $\mathrm{CO}_{2}$ em folhas "de sol" e folhas "de sombra", desenvolvido por Boote \& Jones (1987), que considera o coeficiente de extinção luminosa (k), a reflexão e a transmissão de luz pelo dossel. Assim, um IAF de sol é calculado analiticamente a partir do IAF total e do $\mathrm{k}$ (equação 1), enquanto um IAF de sombra é obtido subtraindo-se o IAF de sol do IAF total (equação 2).

$$
\begin{aligned}
& \operatorname{IAF}_{\text {sol }}=(1 / \mathrm{k})\left[1-\exp \left(-\mathrm{k}^{*} \operatorname{IAF}_{\text {total }}\right)\right] \\
& \mathrm{IAF}_{\text {somb }}=\mathrm{IAF}_{\text {total }}-\mathrm{IAF}_{\text {sol }}
\end{aligned}
$$

A assimilação de $\mathrm{CO}_{2}$ pelas folhas de sol foi calculada por uma curva de resposta exponencial negativa, na qual a assíntota corresponde à fotossíntese máxima de folhas, a 
inclinação inicial representa a eficiência de utilização de luze a variável de entrada é o valor da irradiância fotossinteticamente ativa absorvida (k(1- $\sigma)$ RFA), de acordo com a equação 3 :

$\mathrm{F}_{\mathrm{sol}}=\mathrm{F}_{\max }\left\{1-\exp \left[-\mathrm{Q}_{\mathrm{e}}{ }^{*} \mathrm{k}(1-\sigma) \mathrm{RFA} / \mathrm{F}_{\max }\right]\right\}$

em que $\mathrm{F}_{\max }=$ fotossíntese máxima de folhas individuais $\left(\mu \mathrm{mol}\right.$ de $\left.\mathrm{CO}_{2} \mathrm{~m}^{-2} \mathrm{~s}^{-1}\right) ; \mathrm{Q}_{\mathrm{e}}=$ eficiência de utilização de luz $\left(\mu \mathrm{mol}\right.$ de $\mathrm{CO}_{2} \mathrm{~m}^{-2} \mathrm{~s}^{-1} / \mu \mathrm{mol}$ fótons $\left.\mathrm{m}^{-2} \mathrm{~s}^{-1}\right)$; RFA = radiação fotossinteticamente ativa ( $\mu \mathrm{mol}$ fótons $\mathrm{m}^{-2} \mathrm{~s}^{-1}$ ); $\sigma=$ coeficiente de reflexão e transmissão. A RFA incidente para a fotossíntese de folhas sombreadas, considerando o mesmo k e a mesma eficiência no uso da luz refletida e transmitida por todas as folhas sombreadas, foi determinada pela equação 4 :

$\mathrm{RFA}_{\text {somb }}=\sigma * \mathrm{RFA}\left[1-\exp \left(-\mathrm{k}^{*} \mathrm{IAF}_{\text {somb }}\right)\right] / \mathrm{IAF}_{\text {somb }}$

em que: $\mathrm{RFA}_{\text {somb }}=$ radiação fotossinteticamente ativa para folhas de sombra. Desse modo, a assimilação por folhas de sombra é calculada de modo análogo à de folhas de sol:

$\mathrm{F}_{\text {somb }}=\mathrm{F}_{\max }\left[1-\exp \left(-\mathrm{Q}_{\mathrm{e}}{ }^{*} \mathrm{RFA} \mathrm{A}_{\text {somb }} / \mathrm{F}_{\max }\right)\right]$

em que: $\mathrm{F}_{\text {somb }}=$ assimilação de $\mathrm{CO}_{2}$ por folhas de sombra.

Por fim, a assimilação total do dossel é a soma de ambas as categorias de folhas (equação 6):

$\mathrm{F}_{\mathrm{dos}}=\mathrm{F}_{\mathrm{sol}} * \mathrm{IAF}_{\mathrm{sol}}+\mathrm{F}_{\mathrm{somb}} * \mathrm{IAF}_{\mathrm{somb}}$

em que: $\mathrm{F}_{\mathrm{dos}}=$ assimilação total $\mathrm{CO}_{2}$ pelo dossel.

Utilizando os resultados dessas simulações, foram estimadas as eficiências fotossintéticas dos dosséis. Assumiu-se que Qe para espécies de metabolismo $\mathrm{C}_{4}$ é de aproximadamente 0,054 , independentemente da temperatura ambiente e do regime de luz em que as plantas se desenvolvem (Ehleringer \& Bjorkman, 1977; Dias-Filho, 2002; Ehleringer $\&$ Pearcy, 1983). Também foi assumido um valor de $20 \%$ para o coeficiente de reflexão e transmissão de luz no interior do dossel. O coeficiente de extinção de luz ( $\mathrm{k}$ ) foi calculado nos diferentes pontos do ciclo $\left(\mathrm{k}=-\left[\log _{\mathrm{e}}\left(\mathrm{I} / \mathrm{I}_{\mathrm{o}}\right)\right] / \mathrm{IAF}\right.$, de modo que I e $\mathrm{I}_{\mathrm{o}}$ corresponderam a valores de irradiância abaixo e acima da folhagem, respectivamente). O IAF considerado para a simulação de fotossíntese de dossel foi obtido pelo método não-destrutivo com o analisador de dossel LI-COR, modelo LAI 2000.

Os dados foram avaliados por meio do procedimento GLM do software estatístico SAS (Littel et al., 1996) e a comparação de médias, por meio do comando LSMeans, adotando-se nível de significância de $10 \%$, pelo teste Tukey. Para fotossíntese de folhas individuais, as medições foram feitas em diferentes dias, de acordo com a época, a unidade experimental e o tratamento. Portanto, as medidas foram influenciadas pelo balanço hídrico, pela temperatura do ar e pela umidade relativa. A análise de variância foi feita utilizando-se temperatura de folhas e pressão de vapor d'água como co-variáveis para melhor ajuste do modelo.

\section{Resultados e Discussão}

As estratégias de pastejo ( $(\mathrm{P}=0,0003)$, o ponto do ciclo $(\mathrm{P}<0,0001)$ e a interação estratégias de manejo $\times$ ponto do ciclo $(\mathrm{P}<0,0001)$ afetaram as taxas de fotossíntese de folhas individuais. O ponto do ciclo em que foi realizada a avaliação teve forte impacto sobre as taxas de fotossíntese foliar (Tabela 1), comprovando que, na fase inicial da rebrotação, o potencial fotossintético é pequeno, em média $10,4 \mu \mathrm{mol}$ $\mathrm{CO}_{2} \mathrm{~m}^{-2} \mathrm{~s}^{-1}$. No tratamento $100 \% \mathrm{IL}$, a taxa de fotossíntese foliar pós-pastejo foi de $3,3 \mu \mathrm{mol} \mathrm{CO}_{2} \mathrm{~m}^{-2} \mathrm{~s}^{-1}$, aproximadamente $25 \%$ da média dos outros dois tratamentos. Esse resultado pode ser atribuído ao maior acúmulo de colmos e material morto nesse tratamento e, conseqüentemente, à redução na quantidade de folhas. Além disso, as folhas pós-pastejo ficaram mais tempo sombreadas, em decorrência dos maiores intervalos de pastejo, resultando em menor adaptação à condição de alta luminosidade, o que sugere uma fase inicial mais lenta e, possivelmente, a necessidade da utilização de reservas orgânicas na geração de novos tecidos fotossintetizantes.

O dia médio (DM) foi o ponto do ciclo de rebrotação que, em média, apresentou os maiores valores de fotossíntese foliar, independentemente da estratégia de pastejo. No prépastejo, com a planta em condição fisiológica mais "jovem", o tratamento $95 \%$ IL continuou apresentando os maiores valores de fotossíntese foliar. Enquanto isso, os tratamentos de menor freqüência permaneceram mais tempo em ambientes com maior competição por luz e, conseqüentemente, tiveram seus valores de fotossíntese foliar reduzidos.

Em pastagens de gramíneas de clima temperado, nas quais o IAF é elevado, as folhas que permanecem no dossel após o pastejo não estão adaptadas à alta luminosidade incidente. Em parte, porque foram formadas em condições de baixa luminosidade (Woledge, 1973) ou de alta luminosidade seguida de baixa luminosidade por um período de tempo relativamente longo. Sob lotação intermitente e mesmo sob alta incidência de radiação, o IAF residual apresenta baixa capacidade fotossintética, comprometendo a rebrotação (Grant \& King, 1984).

A taxa fotossintética foliar, após a completa expansão das folhas, decresce com a idade da planta, em decorrência do declínio do potencial metabólico e da mudança de posição relativa da folha dentro do dossel (Acock et al., 1978; Ludlow \& Wilson, 1971; Roux \& Mordelet, 1995). Como 
Tabela 1 - Fotossíntese foliar nos diferentes pontos do ciclo de rebrotação em capim-xaraés submetido a estratégias de pastejo rotativo

Table 1 - Leaf photosynthesis at different moments during the regrowth period in Xaraés palisadegrass pastures as affected by rotational grazing strategies

\begin{tabular}{lcccc}
\hline $\begin{array}{l}\text { Estratégia } \\
\text { Strategy }\end{array}$ & $\begin{array}{c}\text { Pós-pastejo } \\
\text { Post-graze }\end{array}$ & $\begin{array}{c}\text { Dia médio } \\
\text { Midpoint day }\end{array}$ & $\begin{array}{c}\text { Pré-pastejo } \\
\text { Pre-graze }\end{array}$ & $\begin{array}{c}\text { Média } \\
\text { Mean }\end{array}$ \\
\hline $\begin{array}{l}95 \% \text { IL } \\
95 \% \text { LI }\end{array}$ & $14,2 \mathrm{Ba}$ & $24,6 \mathrm{Aa}$ & $26,1 \mathrm{Aa}$ & $21,6 \mathrm{a}$ \\
$\begin{array}{l}100 \% \text { IL } \\
100 \% \mathrm{LI}\end{array}$ & $3,3 \mathrm{Bb}$ & $26,2 \mathrm{Aa}$ & $24,5 \mathrm{Aab}$ & $18,0 \mathrm{~b}$ \\
28 dias & $13,7 \mathrm{Ca}$ & $27,1 \mathrm{Aa}$ & $21,3 \mathrm{Bb}$ & $20,7 \mathrm{a}$ \\
28 days & $10,4 \mathrm{C}$ & $26,0 \mathrm{~A}$ & $24,0 \mathrm{~B}$ & \\
$\begin{array}{l}\text { Média } \\
\text { Mean }\end{array}$ & & & &
\end{tabular}

As médias seguidas de mesma letra minúscula na coluna ou maiúscula na linha não diferem $(P>0,10)$ pelo teste Tukey. Coeficiente de variação $=$ $27,5 \%$

Means followed by the same lower-case letter in columns and the same upper-case letter in the row do not differ by Tukey test at $10 \%$. Coefficient of variation $=27.5 \%$

resultado, quando o IAF aumenta, o potencial fotossintético das folhas, de modo geral, diminui durante a rebrotação (King et al., 1979; Peri et al., 2003). Segundo Peri et al. (2003), pastagens de Dactylis glomerata (L.) apresentaram fotossíntese foliar decrescente ao longo do período de rebrotação, de modo que, aos 60 dias de rebrotação, as folhas mais novas completamente expandidas apresentaram $42 \%$ de redução na fotossíntese máxima observada aos 20 dias. Além dos efeitos de sombreamento, de redução na concentração de $\mathrm{N}$ e de queda na condutância estomática, os autores observaram que as folhas mais novas completamente expandidas tiveram sua idade fisiológica aumentada ao longo da rebrotação, entre outras razões, pela elevação da bainha foliar, de modo que a folha mais nova completamente expandida aos 60 dias foi mais velha que a folha desta mesma categoria aos 20 dias.

Segundo Gomide (2002), à medida que novas folhas e perfilhos jovens surgem em uma pastagem, é estabelecida entre elas uma competição crescente por luz, nutrientes, água, entre outros fatores de crescimento. Em decorrência do auto-sombreamento e do alongamento de colmos, intensifica-se o processo de senescência e morte das folhas mais velhas e até mesmo de perfilhos. Embora Brougham (1956) tenha ilustrado a importância da área foliar residual para a rápida recuperação da pastagem, Brown et al. (1966) consideraram esta variável de pequena importância em virtude de sua baixa eficiência fotossintética. Parsons et al. (1983) observaram que, apesar de as folhas mais novas representarem apenas $42 \%$ da área foliar, foram responsáveis por $77 \%$ da fotossíntese líquida do dossel. Lawlor (1995) ressaltou que a produção de forragem depende mais de fatores determinantes da eficiência de interceptação de luz que da eficiência fotossintética.

Utilizando os resultados de fotossíntese foliar nos diferentes pontos do ciclo de rebrotação, foram feitas estimativas da fotossíntese de dossel (Tabela 2).

De modo geral, o início da rebrotação é caracterizado pela interceptação de aproximadamente $45 \%$ da luz incidente, o que pode ser atribuído, em parte, ao pequeno IAF, caracterizado por folhas normalmente pouco adaptadas às condições de alta luminosidade (Woledge, 1973). Em estratégias de pastejo que permitem intervalos de pastejos muito longos, os valores de fotossíntese foliar são mais baixos no pós-pastejo.

Ao longo do período de rebrotação, com o desenvolvimento das plantas e o conseqüente desenvolvimento do IAF, aumenta-se a quantidade de luz interceptada, incrementando a capacidade fotossintética do dossel (Tabela 2). Em todas as estratégias de pastejo, a fotossíntese máxima foi atingida no dia médio do ciclo, quando o crescimento do dossel ainda não influenciava os padrões de interceptação de luz a ponto de gerar auto-sombreamento significativo. Isso ocorreu no momento do pré-pastejo, quando todos os tratamentos apresentaram menores valores de fotossíntese em comparação aos medidos no DM.

No tratamento de maior freqüência de pastejo $(95 \%$ de IL), o potencial fotossintético do dossel manteve-se com os maiores valores ao longo do ciclo, em decorrência do menor intervalo de pastejo e da manutenção de menor área foliar média (2,08), proporcionando uma arquitetura do dossel que minimizou a competição por luz. Assim, os valores de $\mathrm{k}$ foram sempre menores, com maior penetração de luz no interior do dossel. Sheehy \& Cooper (1973) verificaram que os valores de taxas de crescimento da cultura variaram com a arquitetura do dossel e com o coeficiente de extinção luminosa $(\mathrm{k}=$ - [ log e $\left.\left(\mathrm{I} / \mathrm{I}_{\mathrm{o}}\right)\right] /$ IAF, em que I e $\mathrm{I}_{\mathrm{o}}$ correspondem a valores de irradiância abaixo e acima da folhagem, respectivamente).

O IAF médio foi maior no tratamento $100 \% \operatorname{IL}(2,69) \mathrm{e}$ intermediário no tratamento baseado em calendário $(2,40)$. Esses tratamentos apresentaram períodos de descanso longos o suficiente para aumentar o IAF, gerando no dossel uma competição por luz mais evidente. Isso pode ser comprovado pelo aumento mais acentuado nos valores de $\mathrm{k} \mathrm{e}$, ao mesmo tempo, pelo aumento das quantidades de colmo e material morto. Portanto, o manejo mais adequado foi aquele que proporcionou os maiores valores de fotossíntese de dossel ao longo do ciclo, o tratamento 95\% IL (Tabela 2).

\section{Conclusões}

A taxa fotossintética de folhas individuais reduz quando diminui a freqüência de pastejo, comprometendo o 
Tabela 2 - Índice de área foliar, interceptação luminosa, coeficiente de extinção de luz, fotossíntese foliar e de dossel em capim-xaraés submetido a estratégias de pastejo rotativo em dois ciclos de rebrotação

Table 2 - Leaf area index, light interception, light extinction coefficient, leaf photosynthesis and sward photosynthesis in Xaraés palisadegrass pastures as affected by rotational stocking strategies, in the two cycles where the leaf photosynthesis was measured

\begin{tabular}{|c|c|c|c|c|c|c|}
\hline \multirow[t]{2}{*}{$\begin{array}{l}\text { Estratégia } \\
\text { Strategy }\end{array}$} & \multirow{2}{*}{$\begin{array}{c}\text { Momento do ciclo } \\
\text { Point in cycle } \\
\text { Dia } \\
\text { Day }\end{array}$} & $\begin{array}{l}\text { IAF } \\
\text { LAI }\end{array}$ & $\begin{array}{c}\mathrm{IL} \\
L I\end{array}$ & \multirow{2}{*}{$\begin{array}{c}\mathrm{k} \\
k \\
-\end{array}$} & $\begin{array}{l}\text { Fotossíntese foliar } \\
\text { Leaf photosynthesis }\end{array}$ & $\begin{array}{l}\text { Fotossíntese do dosse } 1^{1} \\
\text { Sward photosynthesis }\end{array}$ \\
\hline & & -- & $\%$ & & $\ldots-\ldots \mu \mathrm{mo}$ & $m^{-2} s^{-1} \ldots \ldots$ \\
\hline \multirow[b]{2}{*}{$\begin{array}{l}95 \% \text { IL } \\
95 \% \text { LI }\end{array}$} & $\begin{array}{l}\text { Pós (1) } \\
\text { Post (1) }\end{array}$ & 0,83 & 46,2 & 0,75 & $14,2 b$ & 12,9 \\
\hline & $\begin{array}{l}\text { DM (11) } \\
\text { MD (11) } \\
\text { Pré (22) } \\
\text { Pre (22) }\end{array}$ & $\begin{array}{l}1,90 \\
3,52\end{array}$ & $\begin{array}{l}75,5 \\
94,5\end{array}$ & $\begin{array}{l}0,74 \\
0,83\end{array}$ & $\begin{array}{l}24,6 \mathrm{a} \\
26,1 \mathrm{a}\end{array}$ & $\begin{array}{l}17,5 \\
13,0\end{array}$ \\
\hline \multirow[b]{2}{*}{$\begin{array}{l}100 \% \text { IL } \\
100 \% \text { LI }\end{array}$} & $\begin{array}{l}\text { Pós (1) } \\
\text { Post (1) }\end{array}$ & 0,93 & 49,5 & 0,74 & $3,3 b$ & 3,3 \\
\hline & $\begin{array}{l}\text { DM (16) } \\
M D(16) \\
\text { Pré (32) } \\
\text { Pre (32) }\end{array}$ & $\begin{array}{l}2,54 \\
4,59\end{array}$ & 97,9 & 0,84 & $\begin{array}{l}26,2 \mathrm{a} \\
24,5 \mathrm{a}\end{array}$ & $\begin{array}{l}15,9 \\
10,2\end{array}$ \\
\hline \multirow[t]{2}{*}{$\begin{array}{l}28 \text { dias } \\
28 \text { days }\end{array}$} & $\begin{array}{l}\mathrm{DM}(14) \\
M D(14)\end{array}$ & 2,66 & 88,4 & 0,81 & $27,1 \mathrm{a}$ & 16,0 \\
\hline & $\begin{array}{l}\text { Pré }(28) \\
\text { Pre (28) }\end{array}$ & 3,78 & 95,4 & 0,82 & $21,3 b$ & 10,9 \\
\hline
\end{tabular}

${ }^{1}$ Valores expressos por unidade de IAF (values expressed per unit of $L A I$ ).

Dentro de tratamentos, médias seguidas da mesma letra minúscula na coluna não diferem $(P>0,10)$ pelo teste Tukey.

Within treatments columns means followed by the same lower-case letter in column do not differ by Tukey test at $10 \%$.

potencial fotossintético do dossel a gerando atraso na rebrotação.

O manejo mais adequado das plantas forrageiras deve priorizar a otimização do uso da luz pelo dossel, resultando em maiores taxas fotossintéticas.

A menor freqüência de desfolhação gera competição por luz e reduz os valores de fotossíntese foliar e de dossel.

O manejo adequado do capim-xaraés deve ser feito com intervalos de pastejo menores que 28 dias, a fim de possibilitar maiores valores de fotossíntese de dossel ao longo do ciclo de rebrotação.

\section{Literatura Citada}

ACOCK, B.; CHARLES-EDWARDS, D.A.; FITTER, D.J. et al. The contribution of leaves from different levels within a tomato crop to canopy net photosynthesis: an experimental examination of two canopy models. Journal of Experimental Botany, v.29, p.815-827, 1978.

BERNARDES, M.S. Fotossíntese no dossel de plantas cultivadas. In: CASTRO, P.R.C.; FERREIRA, S.O.; YAMADA, T. (Eds.). Ecofisiologia da produção agrícola. Piracicaba: Associação Brasileira de Potassa e do Fosfato, 1987. p.13-48.

BOOTE, K.J.; JONES, J.W. Equations to define canopy photosynthesis from quantum efficiency, maximum leaf rate, light extinction, leaf area index, and photon flux density. In: BIGGINS, J. (Ed). Progress in photosynthesis research. The Hague: Martinus Niijhoff Publication, 1987. v.4, p.415-418.
BRAGA, G.J.; PEDREIRA, C.G.S.; HERLING, V.R. et al. Sward structure and herbage yield of rotationally stocked pastures of 'Marandu' palisadegrass [Brachiaria brizantha (A. Rich.) Stapf] as affected by herbage allowance. Scientia Agricola, v.63, n.2, p.121-129, 2006.

BRASIL. Empresa Brasileira de Pesquisa Agropecuária. Sistemas de produção. Disponível em: $<$ http://sistemasdeproducao. cnptia.embrapa.br/FontesHTML/BovinoCorte/BovinoCorteAcre/ index.html $>$. Acesso em: 10/02/05.

BROUGHAM, R.W. Effect of intensity of defoliation on regrowth of pasture. Australian Journal of Agricultural Research, v.7, p.377-387, 1956.

BROWN, R.H. Growth of the green plant. In: TESAR, M.B. (Ed.) Physiological basis of crop growth and development Madison: ASA-CSSA, 1984. p.153-174.

BROWN, R.H.; COOPER, R.B.; BLASER, R.E. Effects of leaf age on efficiency. Crop Science, v.6, p.206-209, 1966.

COOPER, J.P.; WILSON, D. Variation in photosynthetic rate in Lolium. In: INTERNATIONAL GRASSLANDS CONGRESS, 11. 1970, Surfers Paradise. Proceedings... Surfers Paradise: University of Quensland Press, 1970. p.522-527.

DA SILVA, S.C.; PEDREIRA, C.G.S. Princípios de ecologia aplicados ao manejo da pastagem. In: SIMPÓSIO SOBRE ECOSSISTEMAS DE PASTAGENS, 3., 1997, Jaboticabal. Anais... Jaboticabal: FUNEP, 1997. p.1-62.

DIAS-FILHO, M.B. Photosynthetic light response of the $\mathrm{C}_{4}$ grasses Brachiaria brizantha and $B$. humidicola under shade. Scientia Agricola, v.59, n.1, p.65-68, 2002.

EHLERINGER, J.; BJÖRKMAN, O. Quantum yields for $\mathrm{CO}_{2}$ uptake in $\mathrm{C}_{3}$ and $\mathrm{C}_{4}$ plants. Plant Physiology, v.59, p.86-90, 1977.

EHLERINGER, J.; PEARCY, R. Variation in quantum yield for $\mathrm{CO}_{2}$ uptake among $\mathrm{C}_{3}$ and $\mathrm{C}_{4}$ plants. Plant Physiology, v.73, p.555559,1983 
EMPRESA BRASILEIRA DE PESQUISA AGROPECUÁRIA EMBRAPA. Sistema brasileiro de classificação de solos. Brasília, 1999. 412p.

GOMIDE, C.A.M. Morfogênese de cultivares de Panicum maximum (Jacq.). Revista Brasileira de Zootecnia, v.2, p.341-348, 2002.

GRANT, S.A.; KING, J. Grazing management and pasture production: the importance of sward morphological adaptations and morphological photosynthesis. Edinburgh: Hill Farming Research Organization, 1984. p.119-129. (Biannual Report, 1982-83).

JOHNSON, I.R.; PARSONS, A.J.; LUDLOW, M.M. Modeling photosynthesis in monocultures and mixtures. Australian Journal of Plant Physiology, v.16, p.501-516, 1989.

KING, J.; LAMB, W.I.C.; McGREGOR, M.T. Regrowth of ryegrass subject to different cutting regimes and stocking densities. Grass and Forage Science, v.34, p.107-118, 1979.

LAWLOR, D.W. Photosynthesis, productivity and environment. Journal of Experimental Botany, v.46, p.1449-1461, 1995 (Special issue).

LITTELl, R.C.; MILliKen, G.A.; STROUP, W.W. et al. SASâ system for mixed models. Cary: SAS Institute, 1996. 633p.

LOOMIS, R.S.; WILLIAMS, W.A. Productivity and the morphology of crop stands: patterns with leaves. In: EASTIN, J.D.; HASKINS, F.A.; SUlLIVAN, C.Y. et al. (Eds.). Physiological aspects of crop yield. Madison: ASA/CSSA/SSA, 1969. p.27-47.

LUDLOW, M.M.; WILSON, G.L. Photosynthesis of tropical pasture plants. I. Luminance, carbon dioxide concentration, leaf temperature, and leaf-air vapour pressure difference. Australian Journal of Biological Science, v.24, p.449$470,1971$.

PARSONS, A.J.; LEAFE, E.L.; COLLET, B. et al. The physiology of grass production under grazing. I. Characteristics of leaf and canopy photosynthesis of continuously-grazed swards. Journal of Applied Ecology, v.20, p.117-126, 1983.

PEARCE, R.R.; BROWN, R.H.; BLASER, R.E. Relationships between leaf area index, light interception and net photosynthesis in orchardgrass. Crop Science, v.5, p.553-556, 1965.
PERI, P.L.; MOOT, D.J.; McNEIL, D.L. et al. Modelling net photosynthetic rate of field-grown cocksfoot leaves to account for regrowth duration. New Zealand Journal of Agricultural Research, v.46, p.105-115, 2003.

RHODES, I. Relationships between canopy structure and productivity in herbage grasses and its implication for plant breeding. Herbage Abstracts, v.43, p.129-133, 1973.

ROUX, X.L.; MORDELET, P. Leaf and canopy $\mathrm{CO}_{2}$ assimilation in a West African humid savanna during the early growing season. Journal of Tropical Ecology, v.11, p.529-545, 1995.

RYMPH, S.J. Modeling growth and composition of perennial tropical forage grasses. Gainesville: University of Florida 2004. 316p. Thesis (Doctor of Philosophy) - University of Florida, 2004.

SHEEHY, J.E.; COOPER, J.P. Light interception, photosynthetic activity, and crop growth rate in canopies of six temperate forage grasses. Journal of Applied Ecology, v.10, p.239-250, 1973.

VERHAGen, A.M.W.; Wilson, J.H.; BRITTEN, E.J. Plant production in relation to foliage illumination. Annals of Botany, v.27, n.108, p.626-640, 1963.

WELLES, J.M.; NORMAN, J.M. Instrument for indirect measurement of canopy architecture. Agronomy Journal, v. 83, n.5, p.818-825, 1991 .

WOLEDGE, J. The photosynthesis of ryegrass leaves growth in a simulated sward. Annals of Applied Biology, v.73, p.229237, 1973.

ZELITCH, I. The close relationship between net photosynthesis and crop yield. BioScience, v.32, p.796-802, 1982. 